\title{
Three-dimensional Femtosecond Laser Integration in Glasses
}

\author{
Ya CHENG, ${ }^{1}$ Zhizhan XU, ${ }^{1}$ Jian XU, ${ }^{1}$ Koji SUGIOKA, ${ }^{2}$ and Katsumi MIDORIKAWA ${ }^{2}$ \\ ${ }^{1}$ State Key Laboratory of High Field Laser Physics, Shanghai Institute of Optics and Fine Mechanics, \\ P. O. Box 800-211, Shanghai 201800, China \\ ${ }^{2}$ Laser Technology Laboratory, RIKEN-The Institute of Physical and Chemical Research, \\ Hirosawa 2-1, Wako, Saitama 351-0198
}

(Received January 12, 2008)

\begin{abstract}
In the past decade, femtosecond (fs) laser microfabrication has received significant attention because of its powerful three-dimensional (3D) integrating and manufacturing capabilities. A series of multifunctional micro-components and micro-devices, including microoptics, microfluidics, micromechanics, microelectrics, microplasmonics, and so on, can be fabricated and integrated in glass chips with true $3 \mathrm{D}$ configurations using a unified fs laser microprocessing technique. For this reason, this technology is particularly suitable for fabricating lab-on-a-chip (LOC) devices, which have already created a revolution in the fields of chemical, biological, and medical research. A LOC device, due to its highly integration, standardization and automation, allows for performing chemical and biological analyses with ease of use, low sample and reagent consumption, low waste production, high speed of analysis, and high reproducibility. In this paper, we will review the 3D femtosecond laser fabrication of the multifunctional micro-components in glasses as well as their integration. In addition, we will report the latest progress in this active research field, e.g., the fabrication of a tunable microfluidic waveguide, selective surface metallization on dielectrics, and the formation of surface-enhanced-Raman-scattering (SERS) substrate, etc.
\end{abstract}

Key Words: Femtosecond laser microfabrication, Three-dimensional integration, Glass chips

\section{Introduction}

The concept of lab-on-a-chip (LOC) has created a revolution in chemical, biological, and medical sciences. ${ }^{1)}$ A LOC device, which is a palm-sized chip, integrated with functional components such as microfluidics, microoptics, microelectronics, and micromechanics, offers several advantages over the traditional chemical and biological analysis techniques. ${ }^{2)}$ Because of its tiny dimensions, the LOC allows for performing chemical and biological analyses with ease of use, low sample and reagent consumption, low waste production, high speed of analysis, and high reproducibility due to standardization and automation. Recently, progress has been made in integration of 3D photonics and microfluidics for developing optofluidic technology, ${ }^{3,4)}$ which would eventually enable enhanced functionality of the LOC devices. In recent years, femtosecond (fs) laser microfabrication has received significant attention because of its powerful 3D integrating capabilities for LOC device manufacture. ${ }^{5-8)}$ In this paper, we will review the fs laser fabrication of the multifunctional 3D micro-components in glasses as well as their integration. In addition, we will report the latest progress in this active research field, e.g., the fabrication of a tunable microfluidic waveguide, ${ }^{9}$ selective surface metallization on dielectrics, ${ }^{10)}$ and the formation of surface-enhanced-Raman-scattering (SERS) substrate, ${ }^{11)}$ etc.

\section{Experimental}

Some experiments described in this paper were carried out on a commercial fs laser workstation at RIKEN. The laser wavelength, pulse width and repetition rate were $775 \mathrm{~nm}$, $1405 \mathrm{fs}$ and $1 \mathrm{kHz}$, respectively. To ensure a high beam quality, the $6 \mathrm{~mm}$-diameter output laser beam was reduced to $3 \mathrm{~mm}$ by an aperture in front of the focusing system. In most of our experiments, the focusing system was a $20 \mathrm{X}$ microscope objective with a numerical aperture of 0.46 . In these experiments, Foturan glass wafers of $2 \mathrm{~mm}$ thickness were cut into $5 \mathrm{~mm} \times 10 \mathrm{~mm}$ coupons before use. Sample under fabrication was translated by a PC controlled XYZ stage at a resolution of $0.5 \mu \mathrm{m}$. The fabrication process was displayed on the PC monitor by a charge coupled device (CCD).

Other recent experiments mentioned in this paper were performed at a home-built fs laser microfabrication system at SIOM, as illustrated in Fig. 1. The laser wavelength, pulse width and repetition rate were $800 \mathrm{~nm}, 405 \mathrm{fs}$ and $1 \mathrm{kHz}$, respectively. Both microscope cover glass slides and silver-ion-doped glass samples were used in addition to the commercially available Foturan glass for fabricating the microstructures either on the glass surface or its interior.

For the fabrication of microfluidic and microoptical structures inside Foturan glass, after the exposure step, the sample was subjected to a programmed post annealing. First, the temperature was ramped from room temperature to $500{ }^{\circ} \mathrm{C}$ at $5^{\circ} \mathrm{C} / \mathrm{min}$ and held at this temperature for one hour; then it was raised to $605{ }^{\circ} \mathrm{C}$ at $3{ }^{\circ} \mathrm{C} / \mathrm{min}$ and again held for another hour. After the sample cooled down to the room temperature, it was soaked in a $10 \% \mathrm{HF}$ solution to take an ultrasonic bath with variable period of etching time, depending on the size of the hollow structures. Lastly the sample was cleaned in distilled water and dried by a nitrogen gas flow. 


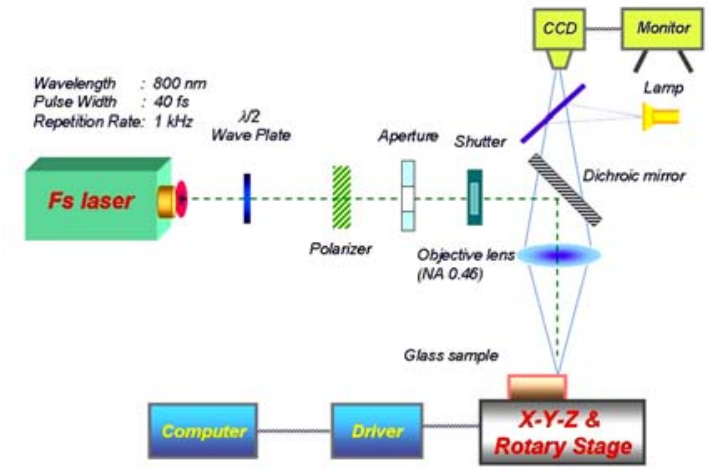

Fig.1. Layout of 3D fs laser micromachining system.

\section{Microcomponents and their 3D Integration}

3.1 Microfluidics and microoptics components

As a first step toward integrating photonics and microfluidics in Foturan glass with true 3D configurations by fs laser microfabrication, we constructed a microchemical reactor composed of microfluidic channels and reservoirs inside the glass. ${ }^{12)}$ Moreover, we showed that freely moving micromechanical elements can be directly fabricated within a hollow chamber. These structures served as microvalves for switching fluid flows between two microfluidic circuits. ${ }^{13)}$ An important issue for 3D laser fabrication is that the axial resolution will always be worse than the lateral one due to the elliptical shape of the focal spot produced by a single objective lens. We have developed two techniques - slit-beam shaping ${ }^{14)}$ and crossedbeam irradiation ${ }^{15)}$ - to form microchannels with circular cross-sections.

Since the microstructuring of Foturan glass by fs laser is a non-ablative processing which results in smooth and debris-free internal surfaces, we realized that not only $3 \mathrm{D}$ microfluidic components, but also 3D microoptics would be able to be fabricated inside the glass. By forming a planar hollow structure vertically buried in the glass, we created a 3D micro-optical mirror that can reflect a beam of light at a right angle by total internal reflection. ${ }^{6}$ The measured reflection loss was only $0.24 \mathrm{~dB}$ at the communication wavelength of $1550 \mathrm{~nm}$. Besides microoptical structures with planar surfaces, microlenses with curved surfaces can also be produced with this technique. ${ }^{16)}$ The microlenses can be important elements for optically based bio-sensor applications, functioning as collimator, focuser, and imaging element.

\subsection{Microfluidic laser}

The 3D photonics and microfluidics can now be readily integrated into a same glass chip for fabricating hybrid devices, such as microfluidic dye lasers which are useful as light sources for optical analyses such as fluorescence detection or photoabsorption spectroscopy in the LOC devices. ${ }^{8)}$

Figure 2a shows the top view of the fabricated microfluidic laser which has an optical microcavity composed of four $45^{\circ}$ micromirrors vertically buried in the glass, a horizontal microfluidic chamber embedded $\sim 400 \mu \mathrm{m}$ below the glass surface, and a microfluidic through channel via the centre of the microchamber. Figure $2 b$ shows a micrograph of the side view of the fabricated microfluidic laser, showing the microchannel with an average diameter of $\sim 80 \mu \mathrm{m}$ and the microchamber with

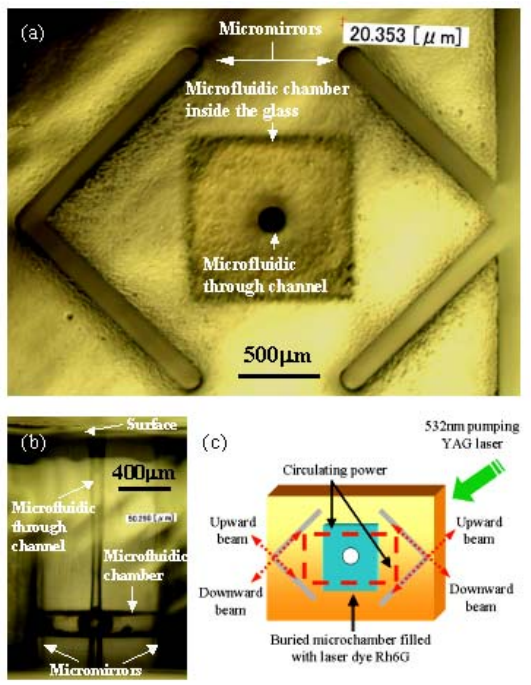

Fig.2. (a) Optical micrograph of the top-view of the microfluidic laser, (b) optical micrograph of the side view of the microfluidic chamber and through channel, and (c) illustration of the light path of the microfluidic laser. ${ }^{8)}$

a thickness of $\sim 200 \mu \mathrm{m}$. Figure 2c illustrates the optical path of the microfluidic laser. The optical cavity is composed of a pair of corner mirrors that are formed by the two micromirrors on the left-hand side and the two on the right-hand side. Light bounces back and forth in the optical cavity by total internal reflections. Lasing action can occur if the microchamber is filled with a gain medium laser dye Rh6G, and then pumped by a frequency-doubled Nd:YAG laser. Since the surfaces of the micromirrors cannot be fabricated perfectly smooth without any roughness, and also the angles between any two micromirrors cannot exactly be at right angles due to the limited precision of fabrication, a small amount of light will eventually leak out from the optical cavity and emit tangentially from the internal surfaces of the micromirrors.

\subsection{Freestanding optical fiber based microdevices}

We also created freestanding optical fibers in glass chip for both guiding light towards a microfluidic cell filled with liquid samples and collecting the light passing through the cell. ${ }^{7)}$ Illustrated in Fig. 3a is a structure composed of a freestanding fiber integrated with a $45^{\circ}$ micromirror at the entrance of the fiber in the glass. The optical path of the coupling scheme is indicated in Fig. 3 a by arrows. The $45^{\circ}$ micromirror allows us to couple the light into the fiber from the side of the sample. Figure $3 \mathrm{~b}$ shows the optical micrograph of the micromirror and entrance of the fiber. The inset of Fig. $3 b$ shows the cross-sectional shape of the fabricated fiber, which has dimensions of about $100 \mu \mathrm{m} \times 80 \mu \mathrm{m}$. Coupling light into the fiber was obtained by focusing a $\mathrm{He}-\mathrm{Ne}$ laser beam at the micromirror using the $20 \times$ objective lens, as shown in Fig. 3c. The guided light was clearly observed at exit of the fiber. The total length of the fiber in Fig. 3c was $8 \mathrm{~mm}$, which is sufficiently long for many microchip applications. The freestanding fibers were incorporated into a microfluidic circuit for biophotonic applications. Shown in Fig. 4a is the 3D schematic view of the integrated structure, namely, a biosensor composed of two series of freestanding fibers intercepted by a 


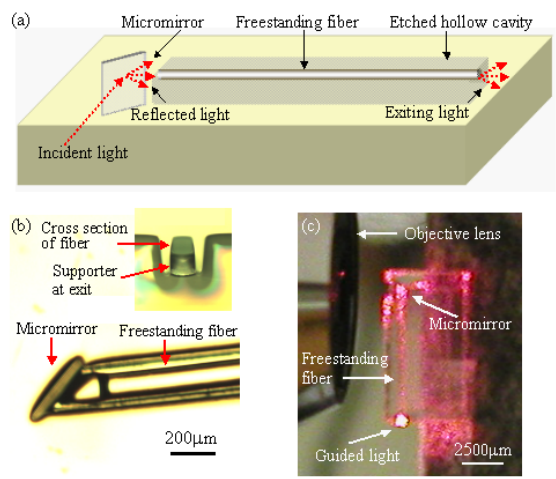

Fig.3. (a) 3D schematic drawing of a freestanding optical fiber integrated with a micromirror fabricated on a glass chip. Red arrows show the optical path of the coupling scheme. (b) Optical micrograph of the top view of the freestanding fiber and the micromirror. The inset (upper right corner) shows the cross-section of the fiber. (c) Digital-camera-captured image of the side coupling of a He-Ne laser beam into the freestanding fiber through the micromirror. ${ }^{7}$

microwell fabricated on the glass chip. Figure $4 \mathrm{~b}$ is the optical micrograph of a part of the fabricated microstructure, showing the fibers and the microwell on the glass surface. To demonstrate that the exiting light from the first fiber can still be coupled into the second fiber, we focused the He-Ne laser beam into the entrance facet of the first fiber by the $20 \times$ objective lens. As shown in Fig. 4c, both scattering light at the microwell and the exiting light at the end of the second fiber can be clearly seen. The coupling loss between the two fibers intercepted by the microwell was about $1 \mathrm{~dB}$.

\subsection{Microfluidic waveguides}

Recently, we fabricated microfluidic channel structures on the surface of borosilicate glass by fs laser direct writing for optical waveguiding application. ${ }^{9)}$ Liquid with a variable refractive index was fed in the microchannels, serving as the cores of the waveguides. We observed the evolution of the transverse modes in the microfluidic waveguides following the change of refractive index of the liquid core using a CCD imaging system. By controlling the refractive index of the

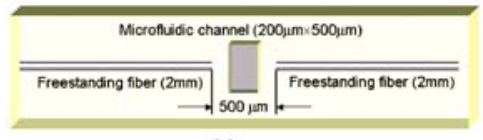

(a)

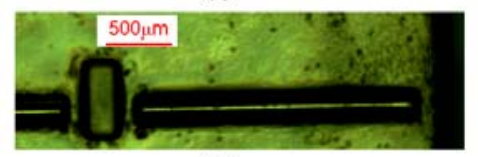

(b)

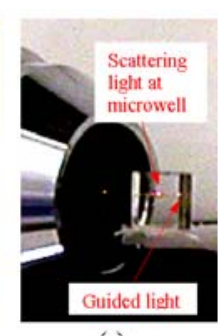

(c)
Fig.4. (a) 3D schematic drawing of two freestanding optical fibers intercepted by a microwell fabricated on a glass chip. (b) Optical micrograph of the structure. (c) Image of the guiding $\mathrm{He}-\mathrm{Ne}$ light through the entire structure. Scattering light at microwell and the guided light at the exit facet of the second fiber are indicated by arrows. ${ }^{7)}$

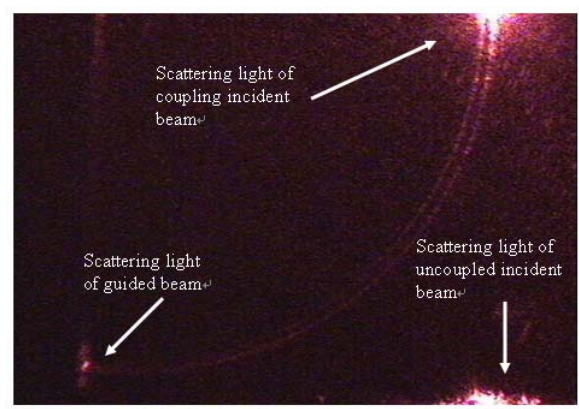

Fig.5. CCD camera captured image of the microfluidic optical waveguide illuminated by He-Ne laser. ${ }^{9)}$

liquid, either a multimode or a single-mode waveguiding can be achieved. The microfluidic waveguide can potentially be used as an important element for biosensor applications.

We examined the waveguiding properties using a He-Ne laser. Figure 5 shows the schematic diagram of the experiment setup. The He-Ne laser beam was first expanded so that it can fill most area of the back aperture of the objective lens. Again, we used a $20 \times$ objective lens to focus the He-Ne beam onto the front end of the microfluidic waveguide. Shown in Fig. 5 is the top view of the waveguide illuminated by the He-Ne laser. It can be seen that the coupled light was confined in the waveguide, and the bright spot at the output end of the waveguide is formed by the scattered light of the outgoing beam. Also can be seen in Fig. 5 is that, most of the scattering light along the waveguide is generated on the sidewalls, whereas little scattering light can be observed from the core of the waveguide. We can evaluate the propagation loss of the waveguide by measuring the attenuation of the scattering light along the waveguide. The measured loss is about $3-5 \mathrm{~dB} / \mathrm{cm}$.

\subsection{Microelectronic components}

For the purpose of developing monolithic and compact chip-sized multifunctional systems, it is desirable to integrate more functions other than the microfluidics and the microoptics in a single glass chip. Therefore, there is an urgent need to incorporate the electrical function into microdevices using fs laser micromachining. Therefore, selective metallization on the surfaces of glasses becomes necessary. ${ }^{10,17)}$

Recently, we have demonstrated the selective metallization on insulator substrates using fs laser direct writing followed by successive electroless copper plating. ${ }^{10)}$ The features of the obtained copper microstructures can be controlled by adjusting the parameters of the laser direct writing and/or the electroless plating process. These metal microstructures show good electrical conductivity and strong adhesion. When the fs laser beam is focused on the glass surfaces coated with silver nitrate

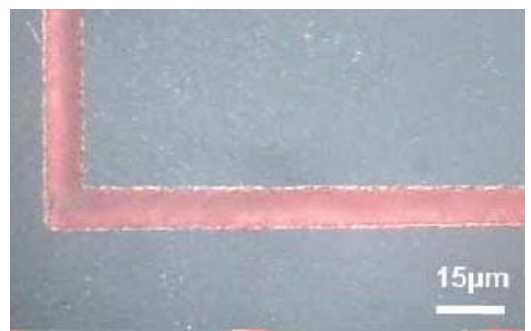

Fig.6. A thin metal line formed on glass surface by fs laser micromachining. ${ }^{10)}$ 
(a)

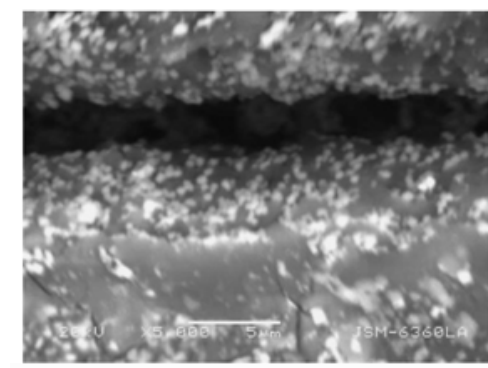

(b)

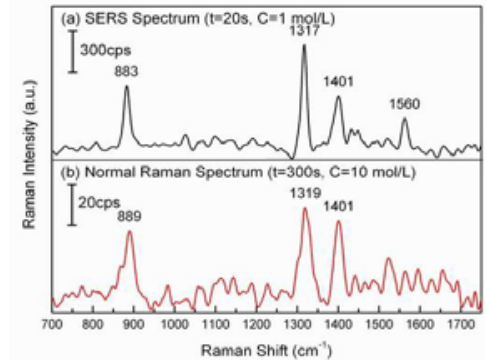

Fig.7. (a) Backscattered SEM image showing silver nanoparticles grown by chemical plating on sidewall of fs-laser-ablated groove. (b) The normal Raman scattering (lower row) and SERS (upper row) spectra of glycine. Note that the integration times, concentrations of sample, and vertical scales are different for the two curves in Fig. 7b. ${ }^{11)}$

films, silver particles can be generated on the irradiated area. In the subsequent electroless plating process, these particles can serve as seeds for in-situ selective copper deposition. Fig. 6 shows optical micrograph of a copper line selectively deposited on the glass surface. One can see that a continuous metal line with smooth edges is formed on the glass substrates. Further development of this technique will provide great potential for rapid and cost-effective fabrication of monolithic 3D micro-electro-opto-fluidic devices.

\subsection{Microplasmonic components}

At present, difficulty in fabricating a stable and reproducible surface-enhanced-Raman-scattering (SERS) substrate bottlenecks it practical application; and incorporating SERS substrate into a micro-sensor device is usually cumbersome and costly. Our recent work shows that a novel SERS substrate with a controllable enhancement factor can be fabricated using fs laser direct writing on silver-ion-doped glass. ${ }^{11)}$ To do so, first, silver atoms were reduced in fs laser ablated area and the subsequent chemical plating promoted the growth of silver nanoparticles, as shown in Fig. 7(a). The high uniformity of the silver nanoparticles ensures a homogeneous enhancement of Raman signal over the fs laser scanned area. As an example, glycine was used as probing molecule to investigate the enhancement effect on the substrate. Raman signals from the normal substrate and the fs laser fabricated SERS substrate are compared in Fig.7 (b). The Raman signal is nearly 2000-fold enhanced by using the SERS substrate. Currently, an effort is made on improvement of the enhancement factor by optimizing the experimental condition. In the future, this technique will be employed to incorporate the Raman substrate into a microfluidic chamber buried in glass chip.
In conclusion, we have demonstrated the $3 \mathrm{D}$ fs laser integration in a single glass chip. The 3D fs laser integration enables an efficient and cost-effective microfabrication free of alignment, fixation, assembly, and packaging, making it possible to manufacture hybrid devices composed of different functional elements. Currently, different approaches to realizing $3 \mathrm{D}$ integration in varies materials based on fs laser microfabrication are under active investigation (such as integration of 3D optical waveguides, ${ }^{18)}$ integration of optics and micromechanics, ${ }^{19)}$ integration of optical waveguides and microfluidic channels, ${ }^{20,21)}$ and so on), heralding the birth of a new research field of science. For integrating a broad variety of functions onto a single chip using fs laser microfabrication, it is important to spend enough effort on the design of the microdevices and the selection of fabrication parameters in order to secure a compatible fabrication process. We have no doubt that the 3D fs laser integration in glass chips will be of great use for LOC application in the future.

The authors are grateful to M. Masuda, K. Obata, and Y. Hanada at RIKEN, as well as H. Sun, Z. Zhou, F. He and Y. Liao at SIOM for their contributions.

\section{References}

1) D. Figeys, and D. Pinto: Anal. Chem. 72 (2000) 330A.

2) M. A. Burns, B. N. Johnson, S. N. Brahmasandra, K. Handique, J. R. Webster, M. Krishnan, T. S. Sammarco, P. M. Man, D. Jones, D. Heldsinger, C. H. Mastrangelo, and D. T. Burke: Science 282 (1998) 484.

3) D. Psaltis, S. R. Quake, and C. Yang: Nature 442 (2006) 381.

4) C. Monat, P. Domachuk, and B. J. Eggleton: Nature Photonics 1 (2007) 106

5) K. Sugioka, Y. Cheng, and K. Midorikawa: Appl. Phys. A 81 (2005) 1.

6) Y. Cheng, K. Sugioka, K. Midorikawa, M. Masuda, K. Toyoda, M. Kawachi, and K. Shihoyama: Opt. Lett. 28 (2003) 1144.

7) Y. Cheng, K. Sugioka, and K. Midorikawa: Opt. Exp. 13 (2005) 7225.

8) Y. Cheng, K. Sugioka, and K. Midorikawa: Opt. Lett. 29 (2004) 2007.

9) H. Sun, F. He, Z. Zhou, Y. Cheng, Z. Xu, K. Sugioka and K. Midorikawa: Opt. Lett. 32 (2007) 1536.

10) J. Xu, Y. Liao, H. Zeng, Z. Zhou, H. Sun, J. Song, X. Wang, Y. Cheng, Z. Xu, K. Sugioka, and K. Midorikawa: Opt. Exp. 15 (2007) 12743.

11) Z. Zhou, J. Xu, Y. Cheng, Z. Xu, K. Sugioka, and K. Midorikawa: Jpn. J. Appl. Phys. A 47 (2008) 189.

12) K. Sugioka, M. Masuda, T. Hongo, Y. Cheng, K. Shihoyama, and K. Midorikawa: Appl. Phys. A 78 (2004) 815.

13) M. Masuda, K. Sugioka, Y. Cheng, T. Hongo, K. Shihoyama, H. Takai, I. Miyamoto, and K. Midorikawa: Appl. Phys. A 78 (2004) 1029.

14) Y. Cheng, K. Sugioka, K. Midorikawa, M. Masuda, K. Toyoda, M. Kawachi, and K. Shihoyama: Opt. Lett. 28 (2003) 55.

15) K. Sugioka, Y. Cheng, K. Midorikawa, F. Takase, and H. Takai: Opt. Lett. 31 (2006) 208.

16) Y. Cheng, H. L. Tsai, K. Sugioka, and K. Midorikawa: Appl. Phys A 85 (2006) 11

17) K. Sugioka, T. Hongo, H. Takai, and K. Midorikawa: Appl. Phys Lett. 86 (2005) 171910

18) W. Watanabe, T. Asano, K. Yamada, K. Itoh, and J. Nishii: Opt. Lett. 28 (2003) 2491.

19) Y. Bellouard, Ali A. Said, and Philippe Bado: Opt. Exp. 13 (2005) 6635

20) R. Osellame, V. Maselli, R. M. Vazquez, R. Ramponi, and G. Cerullo: Appl. Phys. Lett. 90 (2007) 231118.

21) V. Maselli, R. Osellame, G. Cerullo, R. Ramponi, P. Laporta, L. Magagnin, and P. L. Cavallotti: Appl. Phys. Lett. 88 (2006) 191107.

\section{Conclusion}

